\title{
Simulations of Clinical Cases for Learning in e-Health
}

\author{
Nicola Corriero, Pierpaolo Di Bitonto, Teresa Roselli, Veronica Rossano, and Enrica Pesare
}

\begin{abstract}
Over the years, the effectiveness of the learning by doing and the game based learning have been documented by several experimental evidences. In the latest years, the gamification approach has revealed the value of game-based mechanics to create meaningful learning experiences in non gaming systems. For these reasons in the UBICARE (UBIquitous knowledge-oriented HealthCARE) project, aimed at developing an advanced web system that offers different kinds of services to support the management of patients with chronic diseases, the learning component has been realised using both the simulation and the gamification approaches. In particular, the e-learning component starting from real medical records builds on the basis of the user profile a realistic problem, in order to allow specific skills about treatment protocols and diagnostic procedure to be acquired.
\end{abstract}

Index Terms-Simulations, e-learning, e-health, gamification.

\section{INTRODUCTION}

UBICARE (UBIquitous knowledge-oriented HealthCARE) is a project, which aims at designing and building a system that favors the de-hospitalization of patients suffering from peritoneal dialysis and chronic heart failure. In particular, the UBICARE project aims to design and develop a social network framework, which using semantic ontology and knowledge management technologies, support the "Hub and Spoke" health care models. The hub-and-spoke distribution paradigm (or model or network) is a system of connections arranged like a chariot wheel, in which all traffic moves along spokes connected to the hub at the center. The model is becoming popular for hospital networks [1]-[3], where a single specialized center (Hub) is specialized for the treatment of a specific disease and focused on health care, supported by a network of services (Spokes) to transport the patients to reach the minimum severity levels required to take advantage of the specific treatment. In this context, in order to guarantee a high quality service it is necessary that non-specialist medical and paramedical staffs (of Spoke centers) have to be trained on the procedures for diagnosis and patients follow-up.

For these reasons, among the different services offered by

Manuscript received October 20, 2013; revised December 20, 2013. This work was supported in part by the Project UBICARE (UBIquitous knowledge-oriented HealthCARE)-EU-FESR P.O. Puglia Region 2007-2013 Grant in Support of Regional Partnerships for Innovation-Investing in your future (UE-FESR P.O. Regione Puglia 2007-2013 - Asse I - Linea 1.2 - Azione 1.2.4 - Bando Aiuti a Sostegno dei Partenariati Regionali per l'Innovazione - Investiamo nel vostro futuro).

The authors are with Department of Computer Science, University of Bari, Via Orabona, 4, 70125 Bari, Italy (e-mail: nicolacorriero@gmail.com, pierpaolodibitonto@gmail.com, veronica.rossano@uniba.it, enricapesare@gmail.com). the UBICARE system, detailed in [4], [5], the Knowledge Management Service manages all the learning activities and resources in the system. In particular, the services available are the suggestion of personalized learning resources, simulation of clinical cases to train learners to deal with the main problems of the chronic diseases in the ambit of the project, and the management of experience to share information about both official guidelines and medical protocols.

For what concerning the simulation of clinical cases they have been designed and developed using the Gamification approach. "Gamification" is an informal umbrella term for the use of video game elements in non-gaming systems to improve user experience (UX) and user engagement [6]. Gamification can be defined as the use of game mechanics, dynamics, and frameworks to promote desired behaviors into domains like marketing, politics, health and fitness. [7], [8].

The main purpose of this paper is to describe the simulations of clinical cases, which mainly addressed to general practitioners and paramedical staff, which have to be empowered to take care of patients according to standard protocols and to manage any critical state of health of the patient.

The paper is organized as follows: the next sections discusses about the state of the art of gamification in e-health field, Section III describes the simulation of clinical cases in the UBICARE project, Section IV gives an overview of the architecture of the simulation component. Finally some conclusions and future works are discussed.

\section{STATE OF THE ART}

\section{A. Pain Squad Mobile App}

Pain Squad [9] is an iPhone app that helps kids and teens with cancer track how intense their pain is, how long it lasts, where it hurts as well as what helps to treat it. They are also able to record how pain impacts their mood and daily activities, such as doing schoolwork, sleeping and interacting with others.

Cundari created the Pain Squad theme to keep kids engaged and motivated to complete their pain surveys twice a day.

\section{B. Septris}

Septris [10] is web app developed by Stanfor University. This activity provides a practical approach to the identification and application of evidence-based management (best practice) and evidence based guidelines. Case scenarios will be used to put these principles into practice.

Main educational purposes of Septris are:

1) classify epidemiology of sepsis syndrome and 
differentiate between the different forms of sepsis syndromes;

2) integrate best evidence practices, clinical expertise and diagnostic test results for early identification and optimal management of septic states using evidence-based guidelines and clinical decision support tools;

3) demonstrate specific best practice strategies such as fluid resuscitation, early identification with laboratory markers and screening and transfer of patient to higher care with sepsis.

\section{SisterMatch}

SisterMatch is the first matchmaking social game of its kind. By blending a comprehensive matchmaking algorithm with the fun and engagement of social games, SisterMatch aims to bring like-minded women together for support, mentorship and knowledge exchange in a state-of-the-art fashion. Using the metaphor of a community quilt, SisterMatch weaves "Sisters", their stories, opinions, and activities together into a visual tapestry. The matchmaking algorithm helps you find well-matched allies as you make your journey towards thriving with diabetes.

Find and connect with like-minded women for support, mentorship and knowledge exchange relating to diabetes.

\section{Paper to Patient}

Paper to Patient is an interactive educational tool that makes familiarizing yourself with current guidelines on Chronic Obstructive Pulmonary Disease management intuitive and easy. It's also a great way to quickly pinpoint what matters most to you and your patients.

\section{UBIGAME}

"Ubigame" combines the most interesting things of the tools presented to offer an innovative approach to e-learning. It is a $\mathrm{n}$ Elgg [11] plugin in which the user is accompanied in the understanding of the best practices to help patients, caregivers and medical staff to identify the severity of the health problem. In particular, like in "Septris" the evolution of the state of health of the simulated patient will be viewable, through a playful mechanism of rise or fall of the avatar along the screen. By using medical records with real data coming from the social-network UbiCare, and following the approach of "SisterMatch", Ubigame tries to create a community of people with similar health problems. The user is involved in an interactive and fun interface following the pattern "Pain Squad mobile", with the aim to overshadow the problem disease. Finally, from "Paper to Patient" the allocation of points and goals depending on how the user plays has been used.

With respect to the state of the art the innovations introduced in Ubigame are the figure of the coach among the stackholders and the distinction between the roles of the user. In fact, depending on whether s/he is a patient, a caregiver, a nurse or a medical specialist the appropriate therapy will be recommended. The game works different with the different profile. Within the same clinical simulation case the game asks different solutions to different users following own profile.

\section{ARCHITECTURE OF THE SimUlation COMPONENT}

In order to improve usability and maintainability of the component, the user interface and the decisional engine were splitted. A web service was built to retrieve all useful informations for the simulation experience. Web service provides real patient data to help user to learn best practices in real case studies. Web service data are provided by two other web service within the social network UBICARE: (1) the Decision Support Service (DSS) aims at supporting the different users to manage ordinary and extraordinary events. Using data mining algorithms and ontologies the DSS is able to suggest alternative therapies, to send automatic alerts based on patient monitoring, to select interesting case studies, based on real cases, to be used in learning and training processes. The Data Service Gateway (DSG) is a service infrastructure that allows the integration and interoperability of data in the whole system. The component ensures the extensibility of the system, the decoupling between distributed components, the transparency of the communication model and the integration of the system with all different management systems of existing patient records.

Both DSG and DSS web services were built according to the practitioners' instructions. DSS requests to the DSG patient information and according to the received data provides the best action to take to improve the patient's health. Fig. 1 depicts the architecture of the simulation component.

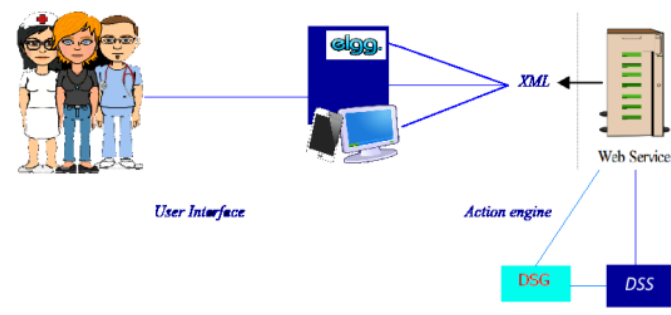

Fig. 1. System components.

All the communications among the different components are done through well-defined protocols. In this way, any modification to the engine of best practices (DSS) or to a particular patient's medical record (DSG) will be available within the simulation component quickly any software updates. Inside the web service are implemented scripts that update daily the engine interrogating other web service platform in order to improve the quality of the game experience.

\section{STAKEHOLDERS}

The simulations are designed for a variety of figures, each with a particular skill. In particular, there are two main categories: the players and the trainers. Among the players there are: caregivers, patients, nurse, general and specialist physicians. The decisions that each professional figure involved in the management of the patient are organized in a pyramidal hierarchy. Thus, each user refers the patient to consulting a higher professional level.

Below is a brief description of the various figures and objectives of the game. 


\section{A. Caregivers and Patients}

Patient and caregiver profile is the lower profile within Ubigame. When a caregiver login to the system, the simulation component tries to learn about simple case studies. The game goal for caregivers is to point out only real problems. In fact a lot of simulated clinical cases end with "Don't worry! Everything it is ok!," Since, usually caregivers and patients are worried about fakes problems, Ubigame aims at helping this kind of people to call a doctor or a medical institute only when necessary. The system, thus, offers learning paths that can be useful in order to face potentially critical situations supporting the patients and their families in making the right choice.

\section{B. Nurses}

Nurse profile is able to identify simple problems, to check therapy and to support patients and caregivers by suggesting who can solve particular problems.

These profiles are the medium level since they can not take decisions about therapy, they have to redirect patients to general or specialist practitioner.

\section{General Practitioner}

General practitioner is asked to solve simple/medium problems according to their skills. General practitioner has access to the standard guidelines about diagnostic and therapeutic protocols in order to support patients even in case of unexpected events. In this way, the patient will refer to the specialist only if necessary. When a general practitioner plays Ubigame, the system aims at supporting them to resolve problems, such as change therapy or recognize critical events by suggesting what the patient can do to solve it.

\section{Specialist Physician}

The specialist physician user profile handles problems with a high level of difficulty.

This kind of user uses the simulation component to verify the system and to indicate possible specialist advice, report therapies.

\section{E. Trainer}

The coach does not play, but trains the system in order to improve and update it. The coach, in fact, creates new problems and associates correct answers to be used by the component to create new simulated clinical cases.

\section{Simulation of Clinical Cases: An Interaction EXAMPLE}

The main screen shows a frame menu with a brief introduction of the game, a summary of the rules, the information in the patient medical record, the patient's monitoring data and the start button game (Fig. 2).

After reading the patient's medical record and the daily monitoring data, as recommended by the game rules, user can start playing.

When the user clicks on the play button, the web service starting from real patient's medical record and the related monitoring parameters, a clinical case is defined according to the user profile and his/her learning needs.
The system provides also the possible interventions that represent the solutions to the patient problem. Interventions can be of three types: Actions, Therapy, and Examinations (Fig. 3).

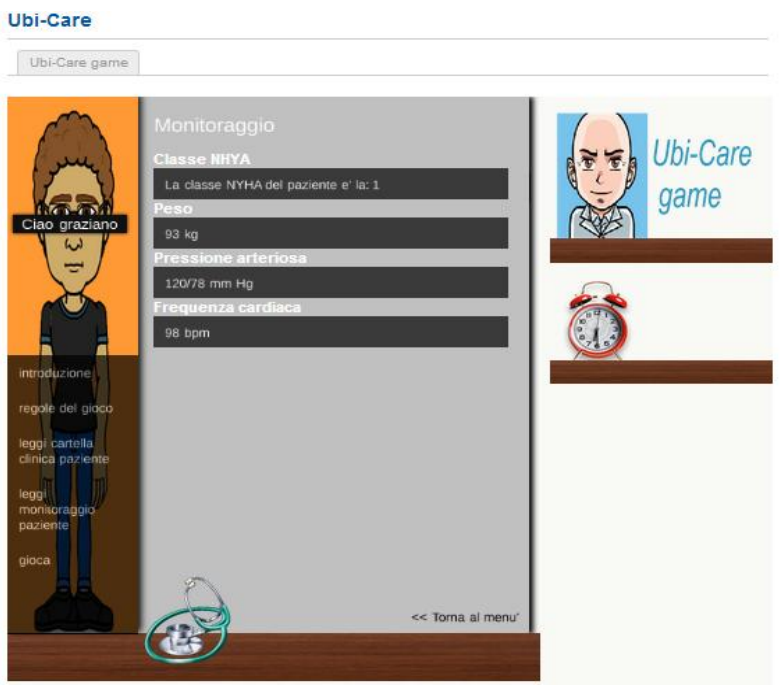

Fig. 2. The main screen of simulation. The monitoring parameters.

Depending on the player profile (patient, nurse, practitioner ...) the interventions change according their knowledge and responsibility.

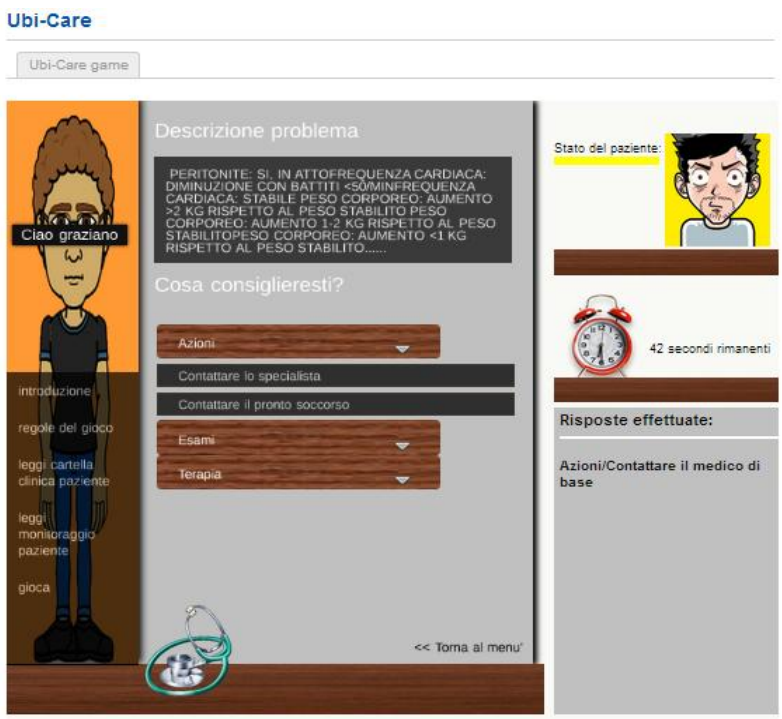

Fig. 3. The patient problem and possible interventions (actions, exams and therapy).

At the same time, when the play button is pressed the countdown starts and the patient represented by an avatar, change her/his health state according to the time and the answers given by the user.

The simulation lasts 90 seconds and with the passing of time if the player does not make the appropriate actions the patient's condition deteriorates. In particular, the avatar of the patient changes color from green to yellow, ending with red and black to represent critical crisis of the patient.

The game ends if the player gives an answer or if the time runs out. Some responses are partially correct or preparatory to the correct one. If one of these is selected, the player is awarded with 5 seconds bonus.

When the simulation ends, a summary supplies detailed information about the game just ended. In particular, a 
detailed feedback is supplied for and against each possible answer. This is particularly useful in case of wrong answers are given during the simulation.

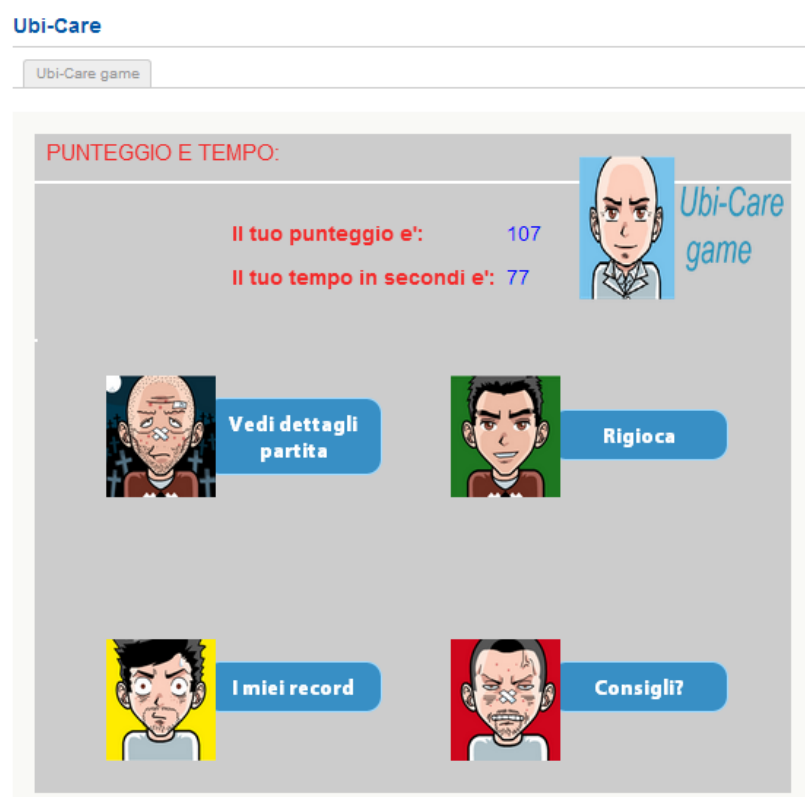

Fig. 4. Post game menu. game details, new game, user statistics, feedback.

Moreover, on the summary screen (Fig. 4) the player can:

1) simulate a new request from a patient dummy;

2) look at the statistics of their play as best score and total games played;

3) obtain feedback to improve understanding of the specific problem simulated in the interaction;

4) send tips to the staff of the game to change some answers or recommend a different attitude of the patient in the game just ended.

The latter feature deserves some explanations. In order to improve the best practices inserted by the trainer and make the system dynamic and adaptive, the medical specialists during the testing phase have asked to add a contact form to send feedback to system/trainer. Usually, in fact, the physicians customize the official protocols and guidelines according to their own experiences.

The whole system is constantly interfaced with the remote web service which is responsible for all data provided to the players. Rules, medical records, monitoring data and the possible answers are all generated in real time from the web service to every request. Moreover, the web service stores all the games played by each player and its related statistics.

\section{CONCluSION}

Learning is an active process and, as all active processes, it requires higher level of motivation to ensure its effectiveness. In the younger students, the motivation to learn is often a native characteristic, but this may decline in older learners. The game is a form of participation and interactive entertainment, as opposed to the more passive activities such as watching television or reading a book.

For this reason, in the UBICARE project the gamification concept has been implied in order to design and build educational games aimed at training general practitioners and paramedical staff to manage daily activities of the patient, the therapy and the critical events. In particular, the simulations interact with the DSS component that supply real clinical cases that the user is asked to resolve.

A first pilot study with a small sample allowed us to investigate the usability of simulations and showed good levels of user satisfaction. Currently, an experiment is ongoing with a sample that involves patients and medical staff at the Polyclinic of Bari. They will be involved in the application of social networking platform UBICARE in the follow-up and treatment of chronic heart failure and peritoneal dialysis to evaluate the performance of the whole system.

\section{REFERENCES}

[1] L. Nobilio and C. Ugolini, "Selective referrals in a 'hub and spoke' institutional setting: the case of coronary angioplasty procedures," Health Policy, vol. 63, no. 1, pp. 95-107, 2003.

[2] J. Stensland, S. M. Speedie, M. Ideker, J. House, and T. Thompson "The relative cost of outpatient telemedicine services," Telemedicine Journal, vol. 5, no. 3, pp. 245-256, 1999.

[3] T. S. W. Lee, E. F. Renaud, and O. F. Hills, "Emergency psychiatry: an emergency treatment hub-and-spoke model for psychiatric emergency services," Psychiatric Services, vol. 54, no. 12, pp. 1590-1594, 2003.

[4] P. D. Bitonto, F. D. Tria, T. Roselli, V. Rossano, and F. Berni, "Distance Education and Social Learning in e-Health," International Journal of Information and Education Technology, vol. 4, no. 1, pp. 71-75, 2014.

[5] F. Berni, N. Corriero, E. Pesare, V. Rossano, and T. Roselli, "A knowledge management service for e-health," International Proceedings of the 6th International Conference of Education, Research and Innovation, Seville, Spain, 2013.

[6] S. Deterding, D. Dixon, L. E. Nacke, K. O'Hara, and M. Sicart, "Gamification: Using Game Design Elements in Non-Gaming Contexts," in Proc. the 2011 Annual Conference Extended Abstracts on Human Factors in Computing Systems, Vancouver, Canada, 2011, pp. 2425-2428.

[7] Gamification by Design: Implementing Game Mechanics in Web and Mobile Apps, 1st ed., Sebastopol, California: O'Reilly Media, pp. xiv.

[8] J. J. Lee and J. Hammer, "Gamification in Education: What, How, Why Bother?” Academic Exchange Quarterly, vol. 15, no. 2, 2011.

[9] Stanford University-Septris Game. [Online]. Available: http://cme.stanford.edu/septris/game/SepsisTetris.html

[10] Pain Squad app. [Online]. Available: http://www.campaignpage.ca/sickkidsapp/files/MediaRelease_Cundar i_PainSquadApp.pdf

[11] Open source framework, ELGG. [Online]. Available: http://elgg.org/

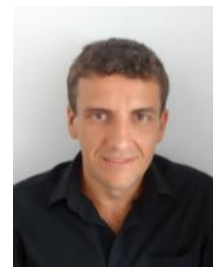

Nicola Corriero received his degree in mathematics in 2004 and informatics in 2007 from University of Bari "Aldo Moro," In 2011, he received the $\mathrm{PhD}$ in informatics from the same University. Currently. He is a research fellow at the National Research Council (CNR). His research interests are embedded system, file system, open source and e-learning.

He was a full professor at University of Vlore (Albania) in 2011-2012 and he taught Computer Systems, Operating Systems and Advanced Data structure

Dr. Corriero is author and/or co-author of over 20 papers published in international journals, books, and conferences and has participated to different research projects aiming at designing and developing innovative solutions in the field of intangible cultural heritage and e-health.

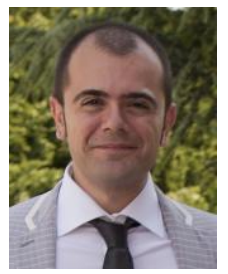

Pierpaolo Di Bitonto has got the bachelor degree in computer science at the University of Bari in October 2002, the master degree in computer science at the University of Bari in March 2004, the PhD in computer science at the University of Bari in May 2009.

He had a Post-Doc fellowship in 2008-2009. His research interests are in, recommender systems and multi-agent system (MAS) particularly in e-learning and tourism. He is co-author of about 40 papers published in journals, conferences and collections (national and international).

Dr. Di Bitonto during the PhD program he addressed theoretical and methodological issues, also designing, developing and testing software tools 
The work has begun with the definition of a multiagent architecture for the student profiling on the basis of the tracking data of the e-learning platform and the learning object personalization. Both the papers presented in the International Workshop on Distance Education Technologies, 2011 and 2012, were awarded of the First place award.

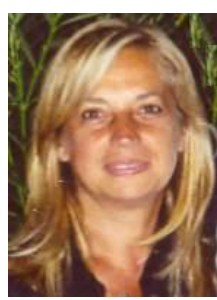

Teresa Roselli has got the degree in computer science (MS) at the University of Bari (Italy) in March 1978 Her research interests are addressed to different e-learning fields both in web and multimedial environments, and in particular to themes such as: the user/student modeling, the interaction of e-learning processes, the sharing of e-learning resources, the multi-agents systems, the semantic web, and recommender systems.

She is an associate professor at the University of Bari "A. Moro" (Italy) since March 2002. She chairs the Multimedial Laboratory, named ADA, of Faculty of Science; she is member of the e-learning Committee of the University of Bari.

Prof. Roselli's efforts have been focused to create relationships among School, University and Business World and, for this purpose, in addition to the activities for the degree courses, she organized in 2001, 2008 and 2012 the Italian Conference on Educational Technology named "DIDAMATICA," She is author of over one hundred of publications including journal and conference papers. She is part of the editorial board of Journal of e-learning and Knowledge Society (Je-LKS), is referee of the International Journal of Distance Education Technologies (IJDET) e per Journal of Educational Technology \& Society. She is part of international and national program committees, and she was general chair of national and international conferences and workshops. Both the papers presented in the International Workshop on Distance Education Technologies, 2011 and 2012, were awarded of the First place award. She was supervisor of different $\mathrm{PhD}$ thesis on e-learning and recommender systems fields.

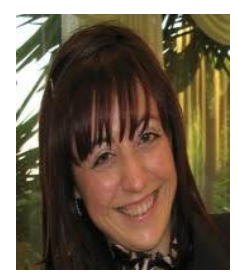

Veronica Rossano has got the degree in computer science (MS) at the University of Bari (Italy) in December 2001 and took the $\mathrm{PhD}$ in informatics at the University of Bari (Italy) discussing a dissertation titled "From Content Objects to Learning Objects: Adding Instructional and Experience-based Information to Educational Metadata" (supervisor prof. Teresa Roselli) in May 2006.

She is an assistant professor at the University of Bari "A. Moro" starting from May 2006. Her research interests are addressed to the e-learning field, and in particular, to the definition of methodologies, specifications and software that can support educational processes and can allow a high level of didactic resource reuse and an adequate level of personalisation of learning path.

Dr. Rossano is author of many journal and conference papers, both at national and international level, and she is part of international and national program committees. She is component of the International Editorial Review Board of: Journal of Distance Education Technologies (IJDET) and Journal of e-learning and Knowledge Society (Je-LKS). Both the papers presented in the International Workshop on Distance Education Technologies, 2011 and 2012, were awarded of the First place award.

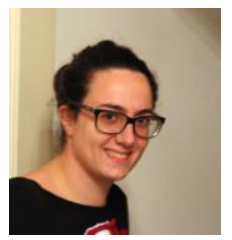

Enrica Pesare has got the bachelor degree in informatics and digital communication in October 2007 and the master degree in computer science in March 2013 with a dissertation on Computational Methods for Interactive Digital Communication entitled "Design and evaluation of a multicriteria method for cultural heritage tourism". She is currently working in the Ubi-Care project for the validation and testing of the components of Knowledge Management Service. 